\title{
La práctica del aborto en manos de feministas en los 70
}

\author{
Raquel Irene Drovetta', 2 (iD 0000-0003-1931-546X \\ 'Consejo Nacional de Investigaciones Científicas y Técnicas, Córdoba, \\ Córdoba, Argentina. 5000 - cct.conicet.cordoba@cordoba-conicet.gov.ar \\ ${ }^{2}$ Universidad Nacional de Villa María, Instituto Académico Pedagógico de Ciencias \\ Sociales, Córdoba, Córdoba, Argentina. 5000 - academica@ics.unvm.edu.ar
}

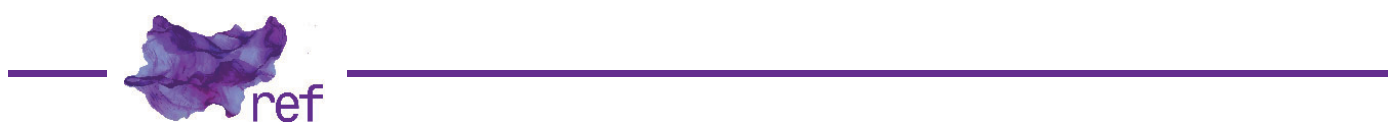

\begin{abstract}
Resumen: Este artículo analiza las principales características de los servicios de aborto llevados adelante por organizaciones feministas en ciudades de Estados Unidos, Italia y Francia entre los años 1969 a 1978, quienes realizaron abortos en la clandestinidad, prescindiendo de la atención de personal médico. Realizamos la reconstrucción histórica y análisis comparativo de los aspectos centrales que caracterizaron a las organizaciones en base a investigaciones científicas, información contenida en artículos de divulgación (radio, periódicos y filmes), testimonios de activistas y documentos de la época actualmente disponibles para su consulta. Entre las conclusiones centrales, destacamos la importancia otorgada al objetivo político que perseguían con la apertura de servicios de abortos, la paulatina evolución de las responsabilidades asumidas por las integrantes de estos espacios y la atención de las mujeres en base a una praxis feminista.
\end{abstract}

Palabras-clave: aborto; feminismos; 1970; organizaciones feministas; método Karman.

The Practice of Abortion in the Hands of Feminists in the 70s

Abstract: The article analyzes the main characteristics of the abortion services performed by feminist organizations in cities of the United States, Italy, and France between 1989 and 1978. These organizations practiced underground abortions, without medical attention. We carried out a historical reconstruction and a comparative analysis of the main aspects of the organizations, based on scientific research, information found on popular science articles (radio, newspaper, and movies), activists' testimonies and documents of that time now available to be consulted. Among the main conclusions, we highlight the importance given to the political objective pursued by opening abortion centers, the slow evolution of the responsibilities taken by their members and the attention to women based on a feminist praxis.

Keywords: Abortion; Feminism; 1970; Feminist organizations; Karman method.

\section{A prática do aborto nas mãos de feministas nos anos 70}

Resumo: Este artigo analisa as principais caraterísticas dos serviços de aborto concretizados por organizações feministas em cidades dos Estados Unidos, da Itália e da França entre os anos 1969 e 1978. As ativistas realizaram abortos na clandestinidade, prescindindo da atenção de pessoal médico. Realizamos a reconstrução histórica e uma análise comparativa dos pontos centrais que caracterizaram às organizações relevadas seguindo investigações científicas, informação contida em artigos de divulgação (rádio, jornais e filmes), testemunhas de ativistas e documentos da época, hoje disponibilizados para consulta. Dentre as conclusões de maior relevância, destacamos o objetivo político que procuravam com a apertura de serviços de abortos, da evolução das responsabilidades aceitadas pelas integrantes desses espaços e da sua atenção às mulheres baseada numa práxis feminista.

Palavras-chave: aborto; feminismo; 1970; organizações feministas; método Karman. 


\section{Introducción}

Desde fines de la década del 60 , diversas organizaciones de mujeres de países industrializados, involucradas en lo que se denominó el feminismo de la segunda ola, profundizaron el trabajo en torno a una agenda de demandas centradas en favor de los derechos reproductivos. Un número importante de estas otorgaron centralidad a la lucha por el aborto legal hasta constituirla en el eje de su trabajo. Ello implicó un alto involucramiento de las activistas con el objetivo de ofrecer a las mujeres que requerían un aborto clandestino una serie de opciones que incluyeron: contactos con médicos prestadores del servicio, la planificación de vuelos chárteres hacia países donde la práctica era legal y, en un número considerable de casos, la realización de abortos en el interior de las propias estructuras de la organización. Paulatinamente, tener abortos sin la intervención de médicos ni médicas se convirtió en una realidad.

La práctica de la interrupción de embarazos realizada por integrantes legas en medicina ha trascendido en la historia. A partir de la búsqueda de literatura especializada, encontramos como característica general que la recuperación de experiencias en países centrales es reciente y ha sido documentada años después de la disolución de los grupos. En este sentido, parece surgir un nuevo interés por conocer detalles vinculados al hacer colectivo en la lucha por el aborto libre.

A los fines de este trabajo, nos concentramos en recuperar, comparar y analizar los antecedentes de organizaciones feministas que ofrecieron el servicio de aborto en contextos de clandestinidad en ciudades de Estados Unidos, Francia e Italia. El período descripto abarca de 1969 a 1978, y coincide con el inicio de sus actividades, hasta su disolución, en paralelo con la sanción de leyes de legalización y despenalización del aborto en cada uno de los tres países.

Llevamos adelante una reconstrucción histórica de los aspectos centrales que caracterizaron a los grupos seleccionados, en base al análisis de datos directos obtenidos de diversas fuentes. En el caso italiano se consultaron los archivos de Radio Radicale, órgano de difusión de información del Partido Radical italiano, que incluyen registros sonoros originales (fundamentalmente entrevistas, grabaciones de discusiones parlamentarias y debates judiciales) y fílmicos que involucraron a ex integrantes de las organizaciones. En el caso francés, se revisaron los registros audiovisuales, filmes documentales y notas de prensa. En el caso estadounidense, se accedió al registro escrito de las entrevistas a ex integrantes de la organización Jane de Chicago (JANE, 1990), así como imágenes y documentos producidos por el grupo y disponibles en el marco del proyecto de archivo documental HerStory de Chicago Women's Liberation Union. Otros datos secundarios fueron obtenidos a partir de publicaciones de divulgación e investigaciones científicas producidas en las últimas cuatro décadas.

La mayor parte de los documentos consultados ha sido recuperada y traducida del inglés, el italiano y el francés, ya que son escasas las referencias a estos grupos producidas en español.

Con los datos reunidos, realizamos un análisis comparativo de los casos entre un conjunto de aspectos específicos, referidos a las prácticas que desarrollaron estos grupos de mujeres en la atención de abortos clandestinos.

Nos interesa recuperar estos hechos con el objetivo de nutrir una genealogía que pueda ser interpretada a la luz de los cambios que atraviesan los movimientos de mujeres en Latinoamérica y el Caribe. En un contexto de emergencia de acciones feministas que promueven el acompañamiento a mujeres que requieren un aborto en un contexto de restricciones legales, creemos que las experiencias de las integrantes de los movimientos de los años 70 pueden ser resignificadas e inspiradoras de nuevas praxis.

\section{Genealogía de tres experiencias \\ El Movimiento de Mujeres de Chicago y el servicio Call Jane}

Una de las experiencias más notables de organizaciones de mujeres desarrolladas en Estados Unidos funcionó en Chicago, Illinois desde fines de los 60 . Se trató de Chicago Women's Liberation Union conocida por sus siglas CWLU, con orientación socialista y feminista. Gran parte de sus integrantes eran mujeres blancas, pertenecientes a la clase media, con estudios universitarios, quienes creían en la igualdad de las mujeres y entendían que esta solo podía lograrse combatiendo al racismo, al clasismo y al sexismo al mismo tiempo (Lara GRIFFIN, 2015; Cara JEPSON, 1999; Chistine RIDDIOUGH, 2015). Quienes formaban parte de este grupo también adherían a los movimientos antibélicos, antimperialistas y al movimiento por los derechos civiles (Chistine RIDDIOUGH, s/d).

1 El acceso a gran parte del material digitalizado fue posible gracias a los servicios de la biblioteca de University of Guelph (ON, Cánada), donde la autora realizó una estancia postdoctoral, con una beca otorgada por el Consejo Nacional de Investigaciones Científicas y Técnicas (CONICET) de Argentina 
En los ocho años que funcionó, CWLU operó con una estrategia fundada en tres pilares: el servicio, la educación y la acción directa; y promovió el trabajo en torno a numerosos intereses que incluyeron la salud de las mujeres y los derechos reproductivos, la educación, los derechos económicos, las artes visuales y la música producida por mujeres, los deportes, la liberación de las lesbianas, la justicia económica, los derechos de las reclusas y la oposición a la guerra (Margareth STROBEL; Sue DAVENPORT, 1999; RIDDIOUGH, 2015; Margareth SCHMID; Cristine RIDDIOUGH, s/d; Mabel BELLUCCI, 2014).

En la práctica, CWLU funcionó como una estructura de paraguas, bajo la que convergieron una amplia gama de proyectos de trabajo, de discusión y de concientización sobre los problemas de la mujer (STROBEL; DAVENPORT, 1999).

Para el mismo período, en Estados Unidos surgieron otras agrupaciones de mujeres en torno a demandas relacionadas con la atención a la salud sexual y reproductiva femenina, que se posicionaron desde un paradigma diferente a la propuesta biomédica hegemónica (Sandra MORGEN, 2002; Carol WEISMAN, 1998). Entre ellas se destaca The Boston Women's Health Collective, quienes presentaron en 1970 su icónica publicación Our Bodies Ourselves. Este libro, convertido en un referente del feminismo estadounidense, puso en palabras el presupuesto sobre el que se asentaron otras agrupaciones feministas: las mujeres se sentían descontentas con "el establecimiento médico capitalista y patriarcal" (BOSTON WOMEN'S HEALTH BOOK COLLECTIVE, 1998, p.136) al que debían recurrir para atender su salud y enfermedad (Amber MUSSER, 2007; Susan WELLS, 2008; Kathy DAVIS, 2007; Sara HAYDEN, 1997).

Comprensiblemente, un área clave de trabajo en CWLU fue la salud. La organización enmarcó el control de la atención médica de las mujeres como parte de un movimiento más amplio hacia la liberación y la política (Jessica JEROME, 2019).

En 1969, algunas de sus integrantes inician un servicio para mujeres que necesitan un aborto, que hará historia.

Formalmente llamado Abortion Counseling Service of Women's Liberation, será conocido como Jane o "el Servicio". Durante los cuatro años que estuvo activo, más de cien mujeres fueron integrantes de este colectivo y se calcula que a través de él se realizaron alrededor de once mil abortos (Laura KAPLAN, 1997; Ruth SURGAL, 1999).

Algunas autoras y documentos de la época sostienen que la relación entre el colectivo Jane y CWLU era de afiliación, lo que implicaba una total autonomía del movimiento e, incluso, Jane podría haber sido creada 5 años antes que CWLU (JEPSON, 1999; HYDE PARK-KENWOOD VOICES, 1973). Por ejemplo, Leslie Reagan (1997) ubica el año de inicio de Jane en 1967, a partir de la iniciativa de Heather Booth, estudiante de la Universidad de Chicago, mientras que el periódico alternativo Hyde Park, en relación con la creación de CWLU, advierte que

[...] si bien decidimos no afiliarnos oficialmente a la Unión, trabajamos en estrecha colaboración organizando grupos de apoyo y trabajando para cambiar la opinión pública sobre el aborto, mientras trabajábamos para que el aborto estuviera disponible (HYDE PARK-KENWOOD VOICES, 1973, p. s/d).

En términos generales, el grupo era heterogéneo e incluía a estudiantes, amas de casa y algunas profesionales. Mientras que la organización central de CWLU definía la membresía al grupo a través de la adhesión al conjunto de principios ideológicos definidos anteriormente, ninguna identidad feminista era necesaria, en cambio, para involucrarse en Jane (Pauline BART, 1987; GRIFFIN, 2015).

El colectivo Jane inicialmente coordinó un servicio en el que ofrecía referencias sobre cómo obtener un aborto facilitando el contacto con los médicos que trabajaban en la clandestinidad. Exintegrantes relatan cómo el contacto entre ellas y las mujeres se realizaba a través del llamado a un número telefónico donde atendía Jane, nombre ficticio que utilizaban las activistas. Allí, comenzaba la búsqueda de un aborto en la clandestinidad, lo que equivalía a obtener una intervención en condiciones "cuidadas" y donde, observando ciertas precauciones, se evitaba que las mujeres se vieran expuestas ante la policía.

El aborto debía pagarse y sólo en ocasiones, por la intermediación de las integrantes frente al médico, se lograban descuentos en caso de que las mujeres no pudieran reunir todo el dinero. Las intervenciones se realizaban en hoteles, bajo notables medidas de seguridad que tendían, fundamentalmente, a mantener el anonimato y la seguridad del médico.

Durante los primeros 18 meses de funcionamiento, las responsabilidades de las mujeres en el servicio evolucionaron más allá de la consejería y el otorgamiento de referencias sobre dónde abortar. Paulatinamente las integrantes comenzaron a ofrecer asistencia al médico durante la intervención. Esto hizo posible que se familiarizaran con la técnica y, eventualmente, recibieran entrenamiento para realizar las interrupciones de embarazo. De esta manera, el aprendizaje se convirtió en parte del componente político del aborto para las feministas involucradas. Como sostiene Leslie Reagan (1997), aspectos como estos permiten ver que Jane era tanto un servicio de salud, como un proyecto de educación política. 
Tiempo después de iniciar las actividades, integrantes de Jane descubren que el médico en realidad no era un profesional de la salud habilitado, lo que finalmente las animó a prescindir de él y realizar ellas mismas los abortos. A inicios de 1971 comenzaron a ejecutar todo el procedimiento de dilatación y curetaje y se convirtieron en proveedoras del servicio de aborto en el primer trimestre y aplicaban otras técnicas para iniciar abortos de segundo trimestre que se completaban en el hospital (BART, 1987). También tomaron en sus manos la decisión acerca de dónde se realizarían los abortos, ofreciendo sus propias casas o rentando apartamentos temporales (KAPLAN, 1997).

En sus memorias, integrantes del servicio reflexionan

[...] claramente, aprender a hacer un DyC [acrónimo de procedimiento dilatación y curetaje] nosotras mismas, de principio a fin, fue el último paso para tener un servicio que pudiera ser controlado y administrado por mujeres (HYDE PARK-KENWOOD VOICES, 1973, p. s/d).

El cambio de paradigma fue notable. Informar a las mujeres acerca de lo que acontecería y hacerlas partícipes activas del evento dejaba en evidencia la notable distancia existente entre las prácticas de Jane y el rol convencional que cumplían las pacientes en el sistema de salud. Cuando las mujeres estaban relajadas y en compañía, el aborto era un procedimiento menos incómodo (KAPLAN, 1997). Incluso, durante las sesiones informativas previas, las integrantes remarcaban a las mujeres su poder de agencia y de decisión (GRIFFIN, 2015).

A esta altura, la organización era autosustentable, lo que permitió mayor dedicación de al servicio, evitando tener que destinar energías en la búsqueda de fuentes externas de sostenimientos (BART, 1987). El precio promedio de un aborto se redujo, pero, aun así, muchas mujeres no podían pagar esa cifra y entregaban la cantidad que podían reunir.

Las integrantes siguieron activas hasta la legalización del aborto en Estado Unidos en 1973. Sin embargo, la detención de siete de ellas en mayo de 1972 contribuyó a acelerar el proceso de desintegración. Finalmente, el grupo se disolvió y parte de las mujeres que trabajaron en Jane continuaron participando de otros proyectos de acción feministas, vinculados, fundamentalmente, con la defensa de los derechos sexuales y reproductivos. Por ejemplo, después de que se legalizó el aborto, se formó el Servicio de Educación y Referencia de Salud de CWLU para guiar a las mujeres a clínicas seguras y respetuosas (JEPSON, 1999).

\section{La experiencia de las feministas en Francia}

El Mouvement pour la Liberté de l'Avortement et de la Contraception, conocido por sus siglas MLAC, inició sus actividades en varias ciudades de Francia en abril de 1973, impulsado por los aires insuflados durante el mayo francés. En la carta de presentación del Movimiento se plasman dos expresiones que serán pilares de la Ley Veil de 1975 que legalizaría el aborto en Francia: la decisión pertenece a la mujer y el aborto es un acto médico (Michelle ZANCARINIFOURNEL 2003, p. 244).

Desde sus inicios, se constituyó como un movimiento mixto, que promovía un cambio profundo en las prácticas de los movimientos sociales hasta ese momento. Fue creado por la iniciativa conjunta de médicos, activistas feministas y militantes de izquierda, quienes articularon redes de colaboración para que las mujeres pudieran realizarse abortos clandestinos. Aun cuando no fue fundado exclusivamente por líderes feministas, estas fueron un componente central de la asociación (Bibia PAVARD, 2012).

EI MLAC se constituyó como una organización nacional que funcionó de manera desagregada, a partir de la cual fueron surgiendo diversos colectivos en los barrios, los hospitales y las universidades de Francia. La diversidad de grupos que se congregaron en torno a este espacio y los múltiples modos de participación habilitados fueron notables.

Los núcleos militantes se establecieron en base a estructuras relativamente autónomas, con la condición de que respetaran la carta fundacional del movimiento (ZANCARINI-FOURNEL, 2003). Aunque solo algunos grupos practicaban abortos, en todos estos ámbitos se discutían aspectos concernientes a la sexualidad y la anticoncepción (PAVARD, 2009). Como muestran las investigaciones citadas, se trataba de una organización legal, que se dedicaba a prácticas ilegales, que incluían tanto la planificación de viajes al exterior para abortar como la realización de abortos en territorio francés.

El avance técnico que significó en Francia la implementación de la técnica Karman para realizar abortos por aspiración, fue el resultado del trabajo vinculado de las redes feministas trasnacionales y su dedicación a promover la circulación de conocimientos. Algunos movimientos europeos y estadounidenses establecieron redes de traducción, apropiación y aplicación de significados, evidenciando lo que Maud Bracke ha mostrado en su obra como "las conexiones trasnacionales del feminismo" específicamente entre Europa y América del Norte (BRACKE, 2015). 
Como resultado de haber propiciado el contacto entre miembros de los círculos de la militancia de izquierda, al inicio la técnica fue implementada por médicos nucleados en torno al Groupe Information Santé (GIS) de París, quienes a partir de 1972 comenzaron a practicar clandestinamente cientos de abortos (ZANCARINI-FOURNEL, 2003).

Desde la perspectiva de Pavard, la estrategia del MLAC de proveer abortos fue pensada como "una acción de protesta sin precedentes" (2009, p. 80), cuyo sentido, sin embargo, no estuvo centrado exclusivamente en la búsqueda por contribuir a la autonomía de las mujeres o en resolver embarazos no planeados de manera segura en la clandestinidad. En todo caso, lograron combinar el interés por mejorar la vida de las mujeres y su emancipación, junto a la búsqueda de la subversión del orden establecido. La autora destaca que se trata de un movimiento que adoptó la realización de abortos clandestinos como una manera de protestar contra la legislación restrictiva del momento y, a la vez, cuestionar a la medicina, a la definición tradicional del activismo y, claramente, a las normas de género (cfr. PAVARD, 2009).

Si bien la asistencia médica al aborto fue una práctica fundante de la organización, la decisión de un grupo de militantes de proveer ellas mismas el servicio y prescindir del control masculino y médico sobre sus cuerpos las condujo a aprender cómo realizar los abortos. De este modo, mujeres de izquierda del MLAC, no médicas, asentadas en Aix-en-Provence, inspiraron con su trabajo el documental Regarde elle a les yeux grand ouverts (presentada en español como Ella tiene los ojos muy abiertos) de 1980, dirigida por Yann Le Masson en Francia. La particularidad del documental es que permite apreciar dos instancias de participación y acompañamiento feminista: un aborto con la técnica Karman, realizado por las activistas con participación de la mujer que abortaba, y partos vaginales sin intervención médica. El filme constituye una alegoría de la trayectoria reproductiva de las mujeres, conformada por abortos y partos, atendida y acompañada exclusivamente por otras mujeres. Se trató de la instalación de una práctica desmedicalizante, que promoviera prescindir de intervención médica y en su lugar estimular el acompañamiento entre las mujeres. ${ }^{2}$ Hacia el final, el documental releva los debates acerca de la disyuntiva que supuso continuar con un servicio de aborto clandestino cuando el aborto en Francia ya estaba legalizado y era ofrecido de manera gratuita por el Estado. Al interior del grupo, prevalecía el interrogante acerca de las consecuencias que supondría la legalización, frente a la reivindicación por la autonomía de las mujeres y su cuerpo.

La llamada Ley Veil, aprobada por el parlamento francés en enero de 1975, despenalizó parcialmente el aborto y a fines de ese año el MLAC se debilitó como movimiento. Sin embargo, como una muestra más de las complejidades en la relación esquiva entre los movimientos sociales y el Estado, en 1977, tras una denuncia del padre de una menor que atravesó un aborto, seis militantes del MLAC fueron procesadas por violar la ley y debieron comparecer ante un tribunal acusadas de intento de aborto, complicidad y práctica ilegal de la medicina (Paul CHOVELON, 1977). Al final, las penas fueron reducidas y, en contrapartida, el movimiento feminista francés se fortaleció, al mismo tiempo que dejó en evidencia las limitaciones de la ley de aborto.

\section{La experiencia italiana de la lucha por el derecho al aborto}

Un número considerable de trabajos se han ocupado de reseñar con detenimiento las características del caso italiano. Entre ellas Maud Anne Bracke, quien describe a los colectivos de mujeres que contribuyeron a inscribir los principios básicos de lo que sería una nueva política del cuerpo, centrada en la autodeterminación para las mujeres en la ltalia de los años sesenta y setenta (BRACKE, 2017). Por ejemplo, el lema "Io sono mia" representó la demanda por el derecho al aborto, al mismo tiempo que la noción de "libertà femminile" se consolidó como una categoría central en el discurso feminista italiano. La demanda que concentró mayor apoyo fue la del aborto libre, gratuito y asistido.

Entre otras organizaciones activas se destacó el Movimento di Liberazione della Donna, conocido como MLD, que se estableció como una afiliación autónoma del Partido Radical en 1971. Inicialmente, desde este espacio dispuso proporcionar información sobre cómo contactar a las parteras que realizaban abortos y sobre la alternativa de buscar atención médica en países como Inglaterra u Holanda, donde la práctica era legal. En la búsqueda por generar acciones que facilitaran el acceso a abortos seguros, el Movimento di Liberazione della Donna apoyó en 1973 la creación en Milán del Centro d'informazione sulla sterilizzazione e sull'aborto (en adelante CISA) en la sede del entonces Partido Radical (Adelle FACCIO, 1973, 1978; Emma BONINO, 1990), el partido de la modernidad y el secularismo, asegura Bracke (2017). Para 1975, tanto el Movimento di Liberazione della Donna como CISA se encontraban trabajando conjuntamente bajo la dirección de la activista Adele Faccio.

2 Otra experiencia vinculada a la práctica del aborto clandestino fue reflejada en el filme Histories d'A un documental francés de 1973, dirigido por Charles Belmont y Marielle Issartel, que formó parte del llamado cine militante fuertemente involucrado en el mayo francés. Filmada a iniciativa del GSI y del MLAC, en él se muestra, entre otras cosas, un aborto mediante el método Karman 
El sentido de ofrecer el servicio de abortos ilegales se orientó a constituirse como una acción de desobediencia civil por parte del Partido Radical y "una desobediencia elegida y declarada, no enterrada e impuesta» (CISA; MLD, 1975, p.18) de parte de las feministas del Movimento di Liberazione della Donna.

Poco tiempo después, CISA se constituyó en una estructura extensa, bien establecida y eficiente, cuyos servicios se ampliaron a otras ciudades italianas a través de la creación de clínicas vecinales que ofrecían el servicio de anticoncepción y lo que actualmente se conoce como planificación familiar. Entre los recursos disponibles se incluían a la esterilización y en alguno de los centros se realizaban abortos en manos de médicos, aplicando la técnica Karman, a un bajo costo o un "precio político" negociado por las mujeres del movimiento con los médicos (CISA; MLD, 1975; RADIO RADICALE, 1978; Mateo ANGIOLI, 2010; FACCIO, 1978) o por el precio de "una oferta libre y voluntaria, según sus convicciones y sus posibilidades" (CISA; MLD, 1975, p. 115).

El uso del método Karman es detallado en la publicación "Aborto: facciamolo da noi" de 1975. Allí se describe que el aborto podía ser realizado en presencia de un médico, pero por personal no médico. El texto oficia como un manual, en el que se recomienda, entre otras cosas, la elección de un espacio doméstico, donde el aborto sea precedido por una reunión de algunas horas, en la que se favorezca el encuentro entre mujeres que abortarán con otras que ya lo hicieron, para conocer sus historias y donde fuera posible recibir información sobre el procedimiento, anticoncepción y sexualidad. La compañía y la conversación con pares es pensada como forma de distender y generar confianza. Incluso, se las anima a observar su vagina con el espejo o a colaborar en la introducción de la cánula (CISA; MLD, 1975).

A medida que avanza la década del 70 los grupos se multiplican. Exintegrantes de CISA continúan en Milán sus actividades prestando el servicio de aborto sin ser médicas, en la búsqueda de lograr, además, "incidencia política entre mujeres de clases populares" (Luciana PERCOVICH, 2005, p. 309). Otras activistas abandonaron los consultorios y abrieron sus propios espacios (en Padova, Milán, Trieste) donde pudieron profundizar en la propuesta: prescindir de la atención de médicos, ofrecer reuniones colectivas en lugar de consultas individuales, promover la práctica de la autoayuda y "continuar con las discusiones políticas al interior del grupo, que rechaza convertirse en un servicio social" (PERCOVICH 2005, p. 60).

Durante el mismo período, otros espacios se abrieron en toda Italia orientados a la atención de la salud de las mujeres. Entre estos, se destaca la experiencia desarrollada a partir de 1974 en diversas ciudades del país donde se abrieron los Consultori Autogestiti, espacios pioneros pensados para la atención de la salud de las mujeres. Estos fueron gestionados por feministas y apoyados por profesionales de la salud simpatizantes de la causa, que ofrecieron información sobre métodos anticonceptivos y atención a problemas de salud femenina. En muchos de estos espacios se llevaron a cabo abortos ilegales, junto con una serie de otras actividades, operando como organizaciones clandestinas hasta su institucionalización (Daniela COLOMBO, 1981; BRACKE, 2015). Destaca Luciana Di Lello que en estos espacios.

[...] las mujeres se encuentran, se reúnen, discuten y se confrontan para una maduración común de sus problemas de la maternidad, de la contracepción, del aborto, del control del propio cuerpo y de la sexualidad y para la organización de la lucha contra toda la forma de la propia opresión (1981, p. 29).

A la distancia, los consultorios pueden ser comprendidos como una figura que fue central en la acción feminista italiana de los 70 , en donde se plasmaron la inspiración de los movimientos estadounidenses como los grupos de liberación de la mujer de Los Ángeles, Boston, Nueva York y la práctica de self-help a cuya diseminación contribuyó Our Bodies, Ourselves publicado por el Boston Women's Health Book Collective (1973). El self-help o autoayuda se consolidó como un conjunto de prácticas que permitían a las mujeres explorar su cuerpo y su sexualidad, basándose en la idea de que la liberación política debía estar arraigada en una nueva relación con el cuerpo, lo que implicaba un desafío explícito a la medicina establecida, así como el objetivo de articular formas alternativas de conocimiento (BRACKE, 2015).

Para los grupos feministas locales, este tipo de influencias representó la posibilidad de contar con espacios donde plasmar de creación de un nuevo lenguaje respecto al cuerpo, la sexualidad, el dolor y la alienación (BRACKE, 2017). Los consultorios representaron más que centros de aborto, oficiando también como "puntos de referencia política y de reunión de las mujeres para la construcción de un movimiento unitario, autónomo" (DI LELLO, 1981, p.29).

La Ley 405 emitida en 1975 permitió reglamentar la existencia de los consultorios, devenidos ahora en consultorios familiares, y disponer su sostenimiento por parte de los gobiernos regionales como espacios de atención de la salud de las mujeres, gestionados (en mayor medida) por mujeres.

De manera contemporánea, la Unione Donne Italiane (sus siglas UDI) en 1975 también se une al Movimento di Liberazione della Donna para ofrecer abortos seguros a las mujeres, 
argumentando que, mientras el aborto fuera ilegal, su práctica sería especialmente riesgosa para las mujeres (Wendy POJMANN, 2005). Al mismo tiempo, por fuera del CISA y del Movimento di Liberazione della Donna existieron otras experiencias menos conocidas, en ocasiones resultantes de redes de contactos informales. En este sentido, advierte Colombo que una parte del movimiento feminista llegó a trabajar en grupos pequeños, sueltos e informales a nivel local (1981). Una de estas experiencias es descripta como el servicio de apoyo al aborto llamado Soccorso Rosa, una red de asistencia mutua para la realización de abortos por aspiración, asentada en Roma, donde las interrupciones eran realizadas por integrantes en espacios domésticos y privados, describiendo así a una de las múltiples experiencias que luego se unieron al Comitato Romano per la liberalizzazione dell'Aborto e della Contraccezione (CRAC) (CILUMBRIELLO; COLOMBO, 2001).

En 1978 la Ley N.194 estableció la despenalización del aborto en Italia. Los grupos se disuelven a posteriori de la legalización, a pesar de la lenta implementación de la ley y de que su aplicación en los hospitales con frecuencia requirió, nuevamente, de la intervención de las exintegrantes de las organizaciones, para ofrecer apoyo a mujeres que necesitaban un aborto. Además, la ley fue modificada para agregar una cláusula sobre objeción de conciencia, lo que sumó un nuevo obstáculo a la demanda por la provisión del aborto legal en ltalia (Lesley CALDWELL, 1981; Jaqueline ANDALL, 1994).

\section{Comentarios a las tres experiencias}

\section{Los inicios y el crecimiento de las organizaciones}

Los grupos que hemos descripto comparten una dinámica de crecimiento exponencial en sus actividades, caracterizadas por una breve, pero intensa genealogía. Las prácticas fundacionales describen a un conjunto de mujeres quienes se establecieron en torno a una organización de izquierda preexistente y a partir de allí se ocuparon específicamente de responder a las demandas de mujeres que necesitaban un aborto.

Paulatinamente se observa en las activistas un cambio en el ejercicio de los roles asumidos. Inicialmente se desempeñaron como "pasadoras" de información, actividades en las que debieron poner en juego un cúmulo de capital social, definido como [el conjunto de recursos actuales o potenciales que están ligados a la posesión de una red duradera de relaciones más o menos institucionalizadas de interconocimiento y de inter-reconocimiento" (Pierre BOURDIEU, 1980, p. 2).

La funcionalidad de dicho capital se plasma en un

[...] conjunto de relaciones sociales que un agente puede movilizar en un momento determinado, que le pueden proporcionar un mayor rendimiento del resto de su patrimonio (los demás capitales, económico y cultural especialmente) (Alicia GUTIÉRREZ, 2005, p. 13)

En este sentido, dicho capital incluía la posibilidad de establecer contactos con médicos que realizaban abortos, la planificación de viajes a clínicas del exterior o la búsqueda de espacios físicos seguros donde realizar las intervenciones.

Las activistas advirtieron como con el paso del tiempo fue necesario gestionar los entornos donde se ofrecía atención a mujeres al resguardo y de manera segura, como en Ios Consultori Autogestitti italianos cuya experiencia culmina con la institucionalización como clínicas regionales; o los departamentos donde Jane realizaba los abortos, llamados "el frente". En la etapa final, la realización de los abortos en sí mismo, constituyó el momento cúlmine de un proceso de ordenamiento al interior de cada grupo que requirió adquirir formación técnica específica, e implementarla a través de una particular praxis feminista. Completar este proceso demandó años de trabajo.

Aun cuando las acciones de realización del aborto fueran referidas como "un servicio a las mujeres", constituyeron en sí mismas expresiones políticas. Esto se explica si observamos que, sobre todo en los casos europeos, la realización de abortos va acompañada de la visibilización de actividades, la vinculación con partidos de izquierda, la preparación de marchas y movilizaciones públicas en demanda de la legalización del aborto, entre las acciones principales. Así, la práctica privada y el espacio público se vincularon notablemente en las experiencias relatadas, a excepción de la experiencia de Chicago.

\section{El apoyo social logrado}

Advierte Pavard que la práctica de los abortos permite identificar áreas comunes de acción "entre organizaciones que tienen, a priori, fuertes divergencias estratégicas entre subversión, servicio y acción legal" (PAVARD, 2012, p. 60). Esta idea es esencial para comprender cómo la lucha por el derecho al aborto se constituye en el núcleo en torno al cual militancias, activismos y profesiones heterogéneas se coordinan, posibilitando coaliciones y alianzas. 
En este sentido, la gestión del apoyo social en cada uno de los contextos donde estos grupos funcionaron se convirtió en un insumo importante para lograr legitimidad en sus demandas por parte de cada uno de los espacios. En el caso de Jane es improbable que haya logrado consolidar dichas bases fuera de los ámbitos feministas, debido a las características de su estricto manejo en la clandestinidad. En todo caso, este apoyo se manifestó a través de la construcción de estrechas redes de colaboración con profesionales de la salud, quienes, de manera sigilosa, apoyaron a las integrantes de Jane en la compra de insumos para los abortos, o intercambiaron información específica sobre mujeres que fueron hospitalizadas, etc. Otros hombres ocasionalmente colaboraron con integrantes de Jane (amigos, amantes o maridos) en aspectos operativos como deshacerse de residuos resultantes de las intervenciones (BART, 1987).

El llamado "secreto mejor guardado de Chicago" no recurrió a la opinión pública buscando adhesiones que impulsaran su trabajo o la causa del aborto libre. En todo caso, Jane tendrá su reconocimiento décadas después de su disolución, y servirá de inspiración a nuevas generaciones de activistas por los derechos sexuales y reproductivos.

Los casos europeos, por su parte, develan la compleja red de asociaciones puestas en juego en el contexto post '68. Los partidos de izquierda, los médicos y las feministas conformaron coaliciones con el objetivo de lograr la legalización del aborto. Esta red de actores congregó un capital social sólido que fue utilizado para impactar en la opinión pública, lo que generó una alta visibilidad social de los involucrados.

Para Pavard (2012), "aborto" es un término genérico que abarca una amplia gama de acciones. Esto significa que las organizaciones realizaban un conjunto de actividades que conducían a luchar por el aborto libre y legal en la esfera pública, y no sólo a través de la realización de abortos clandestinos en el ámbito privado. Comunicar lo que se estaba haciendo fue parte central de la estrategia que implicó dar a conocer ante la sociedad que los abortos se estaban realizando.

Específicamente, el movimiento francés se sirvió de la declaración pública de personalidades reconocidas de la cultura y la ciencia para cosechar el apoyo a la causa en la sociedad. A través de la firma del Manifiesto de las 343 (publicado el 5 de abril de 1971 en Le Nouvel Observateur), reconocidas mujeres intelectuales visibilizaron mediante la autodenuncia (el "Yo aborté") la frecuencia de la práctica del aborto y, consecuentemente, la necesidad de que sea libre. Por su parte, en afinidad con la exposición de las famosas mujeres francesas, médicos del Groupe Information Santé impulsaron la misma estrategia en febrero de 1973, dando a conocer un manifiesto firmado por 331 médicos quienes afirmaron haber realizado abortos. Según la perspectiva de sus integrantes, en la publicidad de sus actos radicaba el éxito del movimiento por la demanda de derecho al aborto (PAVARD, 2012).

De manera contemporánea, el movimiento italiano obtuvo una fuerte adhesión de los movimientos de base, a diferencia del caso francés que cosechó mayor apoyo en ámbitos intelectuales. De hecho, Italia produjo un movimiento feminista vibrante y dirigido a las bases, en sintonía con lo que sucedía entre los movimientos que surgieron en Francia, Alemania Occidental o los Estados Unidos, donde sus discursos, agenda y prácticas a menudo estaban influenciados y transnacionalmente interconectados (BRACKE, 2015).

El Movimento di Liberazione della Donna fue el primer grupo en hacer del aborto en Italia un problema político que requería de acción inmediata (POJMAN, 2005). Las mujeres que había abortado con su ayuda podían asumir el significado político de tal hecho y declarar abiertamente el nombre del grupo que lo había realizado, si así lo deseaban (CISA; MLD, 1975). Algunas de las intervenciones fueron dadas a conocer al periodismo y a la policía. La publicidad funcionó como estrategia política y aumentó la presión hacia el gobierno para rever las leyes existentes (POJMAN, 2005; PERCOVICH, 2005; Gianfranco SPADACCIA, 1990).

Al mismo tiempo, sus integrantes condujeron iniciativas como la recolección de firmas para revisar la ley restrictiva, y la recepción y publicidad de cientos de autodenuncias de mujeres y hombres involucrados en abortos. Si bien recurrieron a estrategias similares a otros colectivos, el sentido otorgado a esta estrategia fue diferente a Francia, donde el valor de la autodenuncia se asentaba en el capital cultural que poseían las mujeres que asumieron haber realizado un aborto (BRACKE, 2017). Publicitar las actividades abiertamente ilegales de estos grupos contribuyó a trasmitir el mensaje de que aun cuando la ley prohibía el aborto, era incapaz de detener su práctica.

Un caso emblemático italiano acontece a raíz de una denuncia realizada contra las mujeres que intervinieron en el aborto de una mujer de 17 años, Gigliola Pierobon, quien también fue detenida y acusada en Padova, en 1973 (cuyo caso fue detallado en Gigliola PIEROBON, 1974). El juicio, que fue llevado adelante acompañado de una fuerte participación política femenina, finalizó con el reconocimiento de la culpabilidad de Gigliola, pero con una absolución mediante la fórmula del perdón judicial. Durante una audiencia, algunas mujeres, entre ellas Alma Sabatini, se declararon a sí mismas sujetas del mismo delito cometido por la acusada: haber abortado (Liliana INGARGIOLA; Marialella CUCCHI, 1987). Se inició así la búsqueda de la 
construcción de una autodenuncia extendida y colectiva, que pudiera tener un impacto político mayor que culminó en una lista de 187 mujeres que revelaban haber abortado y de hombres que admitían haber ayudado a abortar, que fue publicada en la prensa del Partido Radical Liberazione (INGARGIOLA; CUCCHI, 1987).

\section{La organización al interior de los grupos}

Otro aspecto interesante para abordar, a partir de una mirada trasversal al interior de los grupos, es su composición. En los casos abordados los agrupamientos se iniciaron como espacios mixtos, donde, a pesar, de la importancia del trabajo impulsado por las feministas, los hombres y las mujeres desarrollaban tareas diferenciadas y ordenadas en base a la división sexual del trabajo. Fueron los hombres quienes inicialmente se ocuparon de realizar los abortos, cuya consecuencia más evidente fue la concentración de conocimientos sobre aspectos médicos, lo que contribuyó a la reproducción de jerarquías y al reparto de tareas diferenciadas según el sexo.

Jane, el servicio de Chicago, ya no fue integrado por hombres luego de la partida del falso médico. En los grupos europeos, por su parte, la presencia de hombres puede explicarse por la íntima vinculación entre el funcionamiento del movimiento de demanda por el derecho al aborto y el partido de izquierda. Este fue el caso del Movimento di Liberazione della Donna en Italia y su relación con el Partido Radical. Con el paso de los años, la composición mixta del grupo fue problematizada por las feministas integrantes del espacio, lo que dio como resultado la modificación del estatuto en 1975, que desde entonces impidió la participación de hombres (INGARGIOLA; CUCCHI, 1987). Las reconstrucciones históricas que se ocuparon del caso italiano traslucen que una de las divisiones conceptuales más profundas entre hombres y mujeres activistas se tradujo en la imposibilidad de trasmitir la experiencia subjetivante femenina como la "dell'aborto fatto da noi", que aleja a los hombres de la real comprensión, aun en términos políticos, de lo que significa el aborto.

Al respecto, Pavard advierte que hombres y mujeres no definían de la misma manera su compromiso con la organización (2012), algo que paulatinamente fue advertido por las integrantes del MLAC francés: mientras los hombres practicaban los abortos, las mujeres realizaban actividades como intermediarias y acompañantes, y se hallaban a disposición para ocuparse de realizar labores más generales.

\section{El manejo de la técnica: la tecnología médica se vincula al activismo}

La técnica actualmente conocida como de aspiración manual al vacío comenzó a ser desarrollada en Estados Unidos a partir de la década del 50, cuando la perfeccionó Harvey Karman, un psicólogo y activista de California (ver detalles en Tanfer TUNC, 2008a). Este procedimiento permitía realizar abortos entre las primeras seis a ocho semanas de embarazo, mediante la aspiración del contenido del útero a través de una cánula conectada a una jeringa. Entre otros beneficios "la anestesia química es reemplazada por la anestesia verbal" (PAVARD, 2009, p. 80). El aporte central de Karman al perfeccionamiento de la técnica consistió en confeccionar cánulas de plástico flexibles, fácilmente esterilizables y reemplazables de forma económica. El plástico reducía el riesgo de daños uterino que podían ocasiones los instrumentos de metal. ${ }^{3}$

Karman, quien durante un tiempo realizó abortos clandestinos, trasmitió sus conocimientos a notables activistas de California como Carol Downer y Evelyn Rothman del Federation of Feminist Health Centers.

Específicamente en agosto de 1972, el psicólogo demostró su método en París, frente a integrantes del grupo médico Groupe Information Santé, quienes lo adoptaron rápidamente. La introducción del método facilitó la convergencia de objetivos y redes de militancia entre dos movimientos a favor del aborto libre: médicos militantes y feministas. La impresión que tienen los médicos franceses que aplican la técnica es la de estar en poder de "un arma revolucionaria que permitiría desafiar las leyes más represivas" (PAVARD, 2012, p.45). Las sensaciones de libertad que relata Pavard en referencia a los médicos integrantes del colectivo francés, la lleva a identificar a ese momento como un punto de inflexión en la lucha por el aborto libre, que "marca el debut de la práctica del aborto como modo de acción" (PAVARD, 2009, p. 81) y a partir de entonces, el aborto se convierte para estos grupos en la práctica activista más relevante.

Al año siguiente, la visita a Italia de algunas exponentes del Mouvement pour la Liberté de l'Avortement et de la Contraception permitió también trasmitir entre las feministas italianas conocimientos sobre el manejo de esta técnica para la realización de abortos tempranos (BRACKE, 2015). Luciana Percovich (2005) también detalla las posteriores visitas de las activistas

3--No se conocía entonces el aborto con medicamentos (o aborto medicamentoso) a través del uso del misoprostol ni la mifepristona y su potencialidad para lograr un aborto seguro, autónomo y de bajo costo. Recién en la década del '80 el misoprostol comenzó a ser conocido entre las mujeres latinoamericanas como un medicamento que permitía abortar. 
californianas a Roma y Milán en 1973 y del Boston Women's Heath Collective en 1975 y su influencia en la internacionalización de conocimientos sobre sexualidad, aborto, y reproducción.

Existe sin embargo escasa información acerca de las técnicas asociadas a abortos en el segundo trimestre. La bibliografía sugiere que las integrantes de Jane utilizaban la Pasta Leunbach (Norman HAIRE, 1931, p. 1434) o la acción de rotura de saco amniótico para generar un aborto que culminara en el hospital (BART, 1987; REAGAN, 1997). En el caso francés, se detalla que la utilización del método Karman se aplica sólo hasta las 8 semanas de embarazo y no hay referencias de cómo se interviene ante gestaciones más avanzadas. Es posible que el método de Dilatación y Curetaje se continuara utilizando para ciertas ocasiones, de manera paralela a la aspiración.

Nuestro interés es mostrar de qué manera el proceso de difusión de estos conocimientos técnicos oficia como un indicador del establecimiento de redes de comunicación entre agrupaciones feministas, como lo ejemplifican los casos francés e italiano. Por esta razón, describir el alcance y la aplicación de una técnica médica también permite conocer los canales de comunicación establecidos para la época entre las organizaciones a quienes estos conocimientos beneficiaron. Es a través de la reconstrucción de la dinámica de circulación de saberes que es posible conocer las redes de contactos, apoyo y sinergia con que trabajaba el activismo feminista de la segunda ola en países industrializados.

En el caso norteamericano, como documenta Tunc (2008b), en 1965 el $71 \%$ de los abortos legales en los Estados Unidos se realizaron mediante el procedimiento quirúrgico de dilatación y curetaje mientras que en 1972, apenas siete años después, aproximadamente el mismo porcentaje $(72,6 \%)$ de abortos legales en ese país se realizaron con una tecnología de aborto completamente nueva: el aspirador eléctrico de vacío, lo que muestra el aval de las entidades médicas estadounidenses dado a esta técnica.

En contraposición, la ausencia de referencias sobre la utilización del método de aspiración en el grupo del Servicio de aborto en Chicago es un interrogante.

Entre las investigaciones relevadas, existen algunas que describen que existió al menos un contacto entre integrantes de Jane con Harvey Karman. Desde diferentes perspectivas, varios autores y autoras (Charles KING, 1993; KAPLAN, 1997; TUNC, 2008a; Malcom POTTS, 2017) señalan que el resultado devino en un episodio confuso, en el que Karman les propuso probar una técnica aún en desarrollo para realizar abortos durante segundo trimestre entre las mujeres que acudían a su servicio. El encuentro culminó con mujeres internadas con graves complicaciones postaborto y clausuró la posibilidad de otras reuniones o colaboraciones.

El escaso o nulo intercambio de las activistas de Chicago con otras organizaciones se evidencia también ante el hecho de que entre 1969 y 1973 Jane utilizó la técnica de dilatación y curetaje para realizar los abortos, mientras que en otros estados como en California, otros grupos ya utilizaban el método de aspiración, e incluso en esa época se comenzó a implementar en Europa. ¿Por qué no usaron esa técnica? ¿Cómo es posible que el método de aspiración llegara antes a Europa que a esta agrupación en el Estado de Illinois? ¿Qué hubiera significado el uso de esa técnica para las activistas? No existen respuestas claras a estos interrogantes. Tunc (2008b) sostiene que Karman era una persona cuestionada en el ámbito médico hegemónico mientras que, paradójicamente, Pavard (2012) advierte que gozaba de cierta popularidad en el ámbito feminista. Estas representaciones acerca del profesional podrían haber incidido en la decisión de las integrantes de Jane de no utilizar su método de aspiración y, en cambio, continuar aplicando los conocimientos de dilatación y curetaje que aprendieron de quien creían que era un médico certificado.

\section{Conclusiones}

Este trabajo ha intentado reconstruir y trasmitir algunas de las ideas centrales que transitaron entre organizaciones de mujeres feministas de países desarrollados que ofrecieron el servicio de aborto. Los motivos en torno a los que se nuclearon las activistas se basaron en que el aborto debía ser entendido como un problema político y por el cual correspondía reclamar al Estado, un problema de clase, ya que afectaba con mayor fuerza a mujeres de sectores vulnerables, y un problema vinculado al control de la autonomía sobre el cuerpo femenino. La multiplicidad de lecturas de un mismo fenómeno contribuyó al surgimiento de movimientos dinámicos y diversos en torno a la lucha por el derecho al aborto.

La frase que titula este trabajo evidencia lo que posiblemente sea la idea más desafiante de la época: mujeres feministas, desprovistas de titulación formal en medicina, realizaron miles de abortos de manera exitosa, visibilizando nuevas formas de pensar a la salud y al cuerpo femenino.

Prescindir de las y los profesionales de la salud persiguió varios objetivos. Uno de ellos fue la reducción del costo monetario del aborto clandestino, de difícil acceso para mujeres de sectores populares. El avance de la tecnología médica también contribuyó a acelerar el 
proceso de suplantación de profesionales por mujeres legas en la práctica del aborto. Con el abandono paulatino de la técnica de aborto quirúrgico de dilatación y curetaje y la adopción de la técnica de aspiración, las intervenciones se tornaron más simples y se redujeron las complicaciones postabortos.

Al mismo tiempo, se abrió la posibilidad de la instalación de una praxis feminista en la forma de ofrecer el servicio de aborto. Durante el procedimiento, las mujeres atendidas en estos contextos recibieron acompañamiento y soporte emocional, con el objetivo de disminuir las incomodidades. El dolor pudo ser manejado a partir de técnicas descriptas como de anestesia verbal que incluían el acompañamiento por parte de una(s) activista(s) y el relato de lo que ocurría a cada momento. Inclusive, las mujeres fueron animadas a participar voluntariamente de las prácticas que acontecían en su cuerpo, observando o interviniendo en el manejo del instrumental durante la aspiración. Para las militantes feministas, realizar los abortos significó poder prescindir de las lógicas biomédicas patriarcales que intervenían en la prestación del servicio. Fueron capaces de implementar una nueva forma de relacionarse con mujeres fuera de la formula médico-paciente convencional y ganar protagonismo asumiendo un rol central y no simplemente como asistentes de los profesionales de la salud.

Es preciso resaltar que hacer abortos en la clandestinidad implicó optar por una estrategia política al interior de las organizaciones militantes, que supuso ir más allá de ofrecer una respuesta puntual a mujeres con embarazo no planeados. En los casos aquí reseñados, los significados otorgados a la realización de abortos clandestinos fueron múltiples y entre ellos destacamos su utilización como herramienta de lucha política por el aborto libre, la exhibición de las inequidades entre hombres y mujeres y el doble estándar con que se juzgan sus conductas, entre otros aspectos.

Los grupos feministas se diluyeron posteriormente a la aprobación de leyes que legalizaron la práctica. Sin embargo, algunas exintegrantes hicieron reapariciones aisladas en ocasiones de que grupos anti derechos avanzaron con campañas para promover acciones contrarias al mantenimiento de la ley en vigencia. En este sentido, la visibilización de estas experiencias, también se propone contribuir a generar conocimientos sobre la autoorganización feminista en un contexto de permanente avance de los sectores neoconservadores que buscan restringir el acceso al efectivo cumplimiento de los derechos sexuales y reproductivos. Casi dos generaciones han pasado desde el inicio de las actividades de las organizaciones estudiadas, y aún es necesario estar alertas ante posibles ataques a los avances logrados en términos de la ampliación de derechos de las mujeres. Al mismo tiempo, los movimientos de mujeres de Latinoamérica continúan aún en la lucha por la legalización y despenalización del aborto.

Este artículo espera contribuir a pensar líneas de trabajo futuras, donde sea posible indagar qué tipo de estrategias se estarían reactualizando en los contextos locales de crecimiento de espacios feministas y reflexionar acerca de las narrativas sobre el aborto que están emergiendo en cada contexto. Nuestra hipótesis nos conduce a pensar que estas organizaciones de los años 70, en países industrializados, inauguraron prácticas feministas vinculadas al aborto que resultaron precursoras para el trabajo actual de las activistas que trabajan por el derecho al aborto, aun desde la clandestinidad, en países del sur global. De este modo, las enseñanzas dejadas por estas experiencias se tornan contemporáneas y actuales en un momento clave para la lucha por el derecho al aborto. Como advierte Kelly Suzanne O'Donnell (2017), las integrantes de Jane y las simpatizantes de la segunda ola feministas, usan la historia de Jane como forma de mantener la relevancia de los mensajes políticos en tiempos de profundos cambios.

\section{Referencias}

ANDALL, Jacqueline. "Abortion, politics and gender in Italy". Parliamentary Affairs, v. 47, n. 2, p. 238-252, 1994. Disponible en https://doi.org/10.1093/oxfordjournals.pa.a052468.

ANGIOLI, Mateo. Un partito atipico: il caso del Partito Radicale. Tesis para obtener el grado de Magister en Ciencias Políticas, Université Libre de Bruxelles, Facoltà di scienze sociali e politiche, 2010. Disponible en https://www.partitoradicale.it/wp-content/uploads/2018/06/2011-libro-Unpartito-atipico-Matteo-Angioli-TESTO.pdf. Acceso el 12/01/2019.

BART, Pauline. "Seizing the means of reproduction: An illegal feminist abortion collective-How and why it worked". Qualitative Sociology, v. 10, n. 4, p. 339-357, 1987.

BELLUCCI, Mabel. Historia de una desobediencia: aborto y feminismo. Ciudad Autónoma de Buenos Aires: Capital Intelectual, 2014.

BONINO, Ema. "L'aborto clandestino, le azioni di disobbedienza civile del PR e del Cisa dei primi anni 70, il processo del 90, la legge 194." Registro de audio, entrevista del 4 octubre de 1990. Material de archivo compilado por Aurelio Aversa, publicado el 22 de enero de 2010. Disponible 
en http://www.radioradicale.it/scheda/295807/laborto-clandestino-le-azioni-di-disobbedienzacivile-del-pr-e-del-cisa-dei-primi-anni. Acceso el 10/02/2018.

BOSTON WOMEN'S HEALTH BOOK COLLECTIVE. Our bodies, ourselves for the new century: A book by and for women. Boston: Touchstone Books, 1998.

BOURDIEU, Pierre. "Le capital social. Notes provisoires". Actes de la Recherche en Sciences Sociales, v. 31, p. 2-3, 1980.

BRACKE, Maud A. "Our Bodies, Ourselves: The Transnational Connections of 1970s Italian and Roman Feminism". Journal of Contemporary History, Great Easton, Essex, v. 50, n. 3, p. 560-580, 2015. Disponible en https://doi.org/10.1177/0022009414552869.

BRACKE, Maud. A. "Feminism, the state, and the centrality of reproduction: abortion struggles in 1970s Italy". Social History, v. 42 n. 4, p. 524-546, 2017. Disponible en https://doi.org/10.1080/03 071022.2017 .1368234$.

CENTRO D'INFORMAZIONE SULLA STERILIZZAZIONE E SULL'ABORTO (CISA); MOVIMENTO DI LIBERAZIONE DELLA DONNA (MDL). Aborto. Facciamolo da noi. Roma: Roberto Napoleone, 1975.

CALDWELL, Lesley. "Abortion in Italy". Feminist Review, v. 7, p. 49-63, 1981. Disponible en http:/l www.jstor.org/stable/1394759.

CHOVELON, Paul. "Jugement modéré pour les six militantes du MLAC accusées d'infraction à la loi Veil". Les Cahiers du GRIF, v. 16, p. 95, 1977. Disponible en www.persee.fr/doc/grif 07706081 1977_num_16_1_1597. Acceso el10/02/2018.

CILUMBRIELLO, Antonietta; COLOMBO, Daniela. "The Fight for Reproductive Rights in Italy". En: KLUGMAN Barbara; BUDLENDER Debbie (Eds.) Advocating for Abortion Access: Eleven Country Studies. Johannesburg: University of the Witwatersrand, 2001, p. 157-172.

COLOMBO, Daniela. "The Italian Feminist Movement". Women's Studies Int. Quart., v. 4, n. 4, p. 461-469, 1981. Disponible en https://doi.org/10.1016/\$0148-0685(81)96008-5.

DAVIS, Kathy. The making of our bodies, ourselves: How feminism travels across borders. Duke: Duke University Press, 2007.

DI LELLO, Luciana. "C.R.A.C.: una proposta politica sui consultori." effe, Archivio storico di efffe, mensile femminista autogestito, v. 22, p. 29, 1981. Disponible en http://www.herstory.it/ coordinamento-romano-per-i-consultori-autogestiti. Acceso el 4/10/2018.

FACCIO, Adelle. "L'aborto, il codice Rocco, la legge 194, il Cisa, il Partito Radicale". Registro de audio, entrevista realizada el 3 de noviembre de 1973. En: AVERSA, Aurelio (Comp.). Disponible en http://www.radioradicale.it/scheda/567834//aborto-il-codice-rocco-la-legge-194-il-cisa-ilpartito-radicale. Acceso el 10/03/2019.

FACCIO, Adelle. "I diritti delle donne, l'aborto, la legge 194, i referendum del Partido Radicale". Registro de audio del 14 de abril de 1978, Archivo del Partido Radical, Milano, Italia. Disponible en www.radioradicale.it/scheda/535252. Acceso el 7/03/2018.

GRIFFIN, Lara. "The Chicago Women's Liberation Union: White Socialist Feminism and Women's Health Organizing in the 1970s". Thesis, BA, Oberlin College, History, 2015. Disponible en https:ll etd. Ohiolink.edul. Acceso el 3/08/2018.

GUTIÉRREZ, Alicia. "Acerca de la noción de capital social como herramienta de análisis. Reflexiones teóricas en torno a un caso empírico". Perspectivas. Sobre la administración, las políticas públicas y el Estado, Comahue, v. 2, n. 2, p. 7- 26, 2005.

HAIRE, Norman. "Surgical Aspect of Birth Control". The Lancet, Letter to de Editor, December 26, p. 1433-1434, 1931.

HAYDEN, Sara. "Re claiming bodies of knowledge: An exploration of the relationship between feminist theorizing and feminine style in the rhetoric of the Boston Women's Health Book Collective". Western Journal of Communication, v. 61, n. 2, p. 127-163, 1997.

HYDE PARK-KENWOOD VOICES. "The Most Remarkable Story Ever Told". Documento electrónico de CWLU Herstory Project, 1973. Disponible en https://www.cwluherstory.org/jane-articles-mediaarticles/the-hyde-park-voices-series-on-iane. Acceso el 12/08/2018. 
JANE. "Just Call Jane". En: GERBER FRIED, Marlene (Eds.). From Abortion to Reproductive Freedom: Transforming a Movement. Boston: South End Press, p. 93-100, 1990.

JEROME, Jessica. "Much More than a Clinic: Chicago's Free Health Centers 1968-1972". Medical Anthropology, julio, p. 1-14, 2019. Disponible en 10.1080/01459740.2019.1633641.

INGARGIOLA, Liliana; CUCCHI, Marialella. "Dialogo sul Movimento di Liberazione della Donna. Entrevista a INGARGIOLA Liliana". En: Memoria, rivista di storia delle donne. ROSEMBERG; SELLIER, (Eds.), 1987. p. 19-20 Disponible en http://notizie.radicali.it/node/5805. Acceso el 12/01/2018.

JEPSON, Cara. "Sister Against the System". Documento electrónico de CWLU Herstory Project, 5 de marzo de 1999. Disponible en http://webtraxstudio.pairserver.com/cwluherstory/CWLUAbout/ abdoc4.html. Acceso el 10/08/2018.

KAPLAN, Laura. The story of Jane: The legendary underground feminist abortion service. Chicago: University of Chicago Press, 1997.

KING, Charles. "Calling Jane". Women \& Health, v. 20, n. 3, p. 75-93, 1993. Disponible en https:ll doi.org/10.1300/J013v20n03 05.

MORGEN, Sandra. Into Our Own Hands. The women's health movement in the United States, 19691990. Unites Estates: Rutgers University Press, 2002.

MUSSER, Amber J. "From Our Body to Yourselves: The Boston Women's Health Book Collective and Changing Notions of Subjectivity 1969-1973". Women's Studies Quarterly, v. 35, n. 1/2, p. 93-109, 2007.

O'DONNELL, Kelly Suzanne. "Reproducing Jane: Abortion Stories and Women's Political Histories". Signs: Journal of Women in Culture and Society, v. 43, n. 1, p. 77-96, 2017.

PAVARD, Bibia. "Genre et militantisme dans le Mouvement pour la liberté de l'avortement et de la contraception. Pratique des avortements (1973-1979)". Clio. Histoire, femmes et sociétés, v. 29, n. 68, p. 79-96, 2009. Disponible en http://journals.openedition.org/clio/9217.

PAVARD, Bibia. "Quand la pratique fait mouvement. La méthode Karman dans les mobilisations pour l'avortement libre et gratuit (1972-1975)". Sociétés contemporaines, vol, 85, n. 1, p. 43-63, 2012. Disponible en https://doi.org/10.3917/soco.085.0043.

PERCOVICH, Luciana. La coscienza nel corpo: donne, salute e medicina negli anni Settanta. Milán: Fundacione Badaracco-Franco Angeli, 2005.

PIEROBON, Gigliola. II processo degli angeli: (storia di un aborto). Roma: Tattilo, 1974.

POJMANN, Wendy. "Emancipation or Liberation? Women's Associations and the Italian Movement". Historian, v. 67, n. 1, p. 73-96, 2005.

POTTS, Malcom. "Karman Canula". Documento electrónico publicado en Museum of Contraception and Abortion, 2017. Disponible en http://en.muvs.org/topic/karman-cannula-en/. Acceso el 22/01/2019.

RADIO RADICALE. "Aborto (le attività del Cisa)". Registro de audio de la audiencia 3 de octubre de 1978, Florencia, Italia. Disponible en https://www.radioradicale.it/scheda/6566/6583-abortole-attivita-del-cisa. Acceso el 2/02/2018.

REAGAN, Leslie. J. When abortion was a crime: Women, medicine, and law in the United States, 1867-1973. California: Univ. of California Press, 1997.

RIDDIOUGH, Chistine. "Our Band of Sisters. Building Culture and Community in the Chicago Women's Liberation Union”. Documento electrónico de CWLU Herstory Project, 2015. Disponible en https://www.cwluherstory.org/our-band-of-sisters/. Acceso el18/08/2018.

RIDDIOUGH, Chistine. "Health Care Organizing in the Chicago Women's Liberation Union". Documento electrónico de CWLU Herstory Project (sin fecha). Disponible en https://www. cwluherstory.org/analysis-articles/health-care-organizing-in-the-chicago-womens-liberationunion. Acceso el 10/03/2018. 
SCHMID, Margareth; RIDDIOUGH, Christine. "Socialist Feminist Theory and Strategy in the Chicago Women's Liberation Union". Documento electrónico de CWLU Herstory Project (sin fecha). Disponible en https://www.cwluherstory.org. Acceso el 10/03/2018.

SPADACCIA, Gianfranco. "Aborto: processo d'appello ad appartenenti al CISA ed al PR". Registro de audio, entrevista de Laura Cesaretti, el 3 de octubre de 1990. Disponible en http://www. radioradicale.it/scheda/3721 1/aborto-processo-dopo-15-anni-ad-appartenenti-al-cisa-ed-al-pr. Acceso el 10/02/2018.

STROBEL, Margareth; DAVENPORT, Sue. "The Chicago Women's Liberation Union: An Introduction by Margaret "Peg" Strobel and Sue Davenport". Documento electrónico de CWLU Herstory Project, 1999. Disponible en http://webtraxstudio.pairserver.com/cwluherstory/CWLUAbout/abdoc 1.html. Acceso el 8/08/2018.

SURGAL, Ruth. "Organizing a Clandestine Abortion Network". Documento electrónico de CWLU Herstory Project, 1999. Disponible en https://www.cwluherstory.org/jane-stories-articles/organizinga-clandestine-abortion-network? ra $=$ Jane $\% 3 A \% 20$. Acceso el 10/08/2018.

TUNC, Tanfer Ermin. "Harvey Karman and the super coil fiasco: A forgotten episode in the history of abortion technology". European Journal of Contraception and Reproductive Health Care, v. 13 , n. 1, p. 4-8, 2008a. Disponible en https://doi.org/10.1080/13625180701746461.

TUNC, Tanfer Ermin. "Designs of devices: The vacuum aspirator and American abortion technology". Dynamis, v. 28, p. 353-376, 2008b.

WEISMAN, Carol. Women's Health Care. Activist Traditions and Institutional Change. Baltimore: The Johns Hopkins University Press, 1998.

WELLS, Susan. "Our bodies, ourselves: Reading the written body". Signs: Journal of Women in Culture and Society, v. 33, n. 3, p. 697-723, 2008.

ZANCARINI-FOURNEL, Michelle. "Histoire(s) du MLAC (1973-1975)". Clio. Histoire, femmes et sociétés, v. 18, p. 241-252, 2003. Disponible en http://journals.openedition.org/clio/624.

Raquel Irene Drovetta (raqueldrovetta@conicet.gov.ar) es Licenciada en Sociología y Doctora en Antropología Social por la Universidad de Buenos Aires. Posee un Posdoctorado en el Instituto de Medicina Social de la Universidad Estadual de Rio de Janeiro. Actualmente es Investigadora del Consejo Nacional de Investigaciones Científicas y Técnicas (CONICET) y Profesora Adjunta en el Departamento de Sociología de la Universidad Nacional de Villa María en Argentina. 


\section{COMO CITAR ESTE ARTÍCULO, DE ACUERDO CON LAS NORMAS DE LA REVISTA}

DROVETTA, Raquel Irene. "La práctica del aborto en manos de feministas en los 70". Revista Estudios Feministas, Florianópolis, v. 29, n. 2, e67176, 2021.

\section{CONTRIBUCIÓN DE AUTORÍA}

No se aplica.

\section{FINANCIACIÓN}

No se aplica.

\section{CONSENTIMIENTO DE USO DE IMAGEN}

No se aplica.

\section{APROBACIÓN DE COMITÉ DE ÉTICA EN INVESTIGACIÓN}

No se aplica.

\section{CONFLICTO DE INTERESES}

No se aplica.

\section{LICENCIA DE USO}

Este artículo tiene la licencia Creative Commons License CC-BY 4.0 International. Con esta licencia puedes compartir, adaptar, crear para cualquier finalidad, siempre y cuando cedas la autoría de la obra.

\section{HISTORIAL}

Recibido el 22/08/2019

Presentado nuevamente el 18/11/2020

Aprobado el 04/12/2020 